\title{
Prevalence and Incidence of Patients with Fibromyalgia in Catalonia between 2010 and 2017. A Descriptive Observational Study
}

Glòria Sauch Valmaña ( $\nabla$ gsauch.cc.ics@gencat.cat)

Unitat de Suport a la Recerca de la Catalunya Central, Fundació Institut Universitari per a la Recerca a l'Atenció

Primària de Salut Jordi Gol i Gurina (IDIAPJGol)

\section{Queralt Miró Catalina}

Unitat de Suport a la Recerca de la Catalunya Central, Fundació Institut Universitari per a la Recerca a l'Atenció

Primària de Salut Jordi Gol i Gurina (IDIAPJGol)

Josep Vidal-Alaball

Unitat de Suport a la Recerca de la Catalunya Central, Fundació Institut Universitari per a la Recerca a l'Atenció Primària de Salut Jordi Gol i Gurina (IDIAPJGol)

\section{Research Article}

Keywords: Fibromyalgia, Incidence, Prevalence, Primary care, Sociodemographic data

Posted Date: September 30th, 2021

DOI: https://doi.org/10.21203/rs.3.rs-753305/v1

License: (c) (i) This work is licensed under a Creative Commons Attribution 4.0 International License. Read Full License 


\section{Abstract}

Background: To measure the prevalence and cumulative incidence of individuals diagnosed with fibromyalgia (FM) in Catalonia between 2010 and 2017.

Methods: A retrospective observational study of the population of Catalonia between 2010 and 2017, both included, was designed to describe the incidence, prevalence and sociodemographic characteristics of individuals diagnosed with fibromyalgia. A total of 56,098 patients were included in the study. The scope of the study were the 283 Primary Care Teams (PCT), all managed by the Instituto Catalán de la Salud [Catalan Institute of Health] (ICS).

Results: The diagnosis of FM is higher in females (95.4\%) than males (4.55\%), with a mean age of 53.0 [45.0-61.0] years. The prevalence of FM in the total population was $0.4 \%$ in 2010 and $1.4 \%$ in 2017 . The highest prevalence was found in the 55-65 age group (1.05\% in 2010, and 2.46\% in 2017). A relationship was found between the prevalence of FM and the degree of socioeconomic deprivation in urban areas: the greater the deprivation, the greater the prevalence of FM. The cumulative incidence of FM in the population remained constant over time $(0.11 \%$ in 2010 and $0.10 \%$ in 2017), being more prevalent in women than men (0.18\% women, $0.01 \%$ men in 2017$)$.

Conclusions: Our study confirms that FM is a prevalent disease in Catalonia, with an upward trend in recent years and it is more prevalent in women.

\section{Introduction}

The World Health Organization (WHO) recognised Fibromyalgia (FM) as a disease in 1992, assigning it the code M79.0 in the International Classification of Diseases manual (ICD-10). FM is a disease with an unknown origin which is characterised by generalized musculoskeletal pain which is usually accompanied by other symptoms [1] such as fatigue [2], disturbed sleep patterns, anxiety, depression and reduced functional capacity [3-5]. It mainly affects women between the ages of 20 and 55 [6], and has a significant impact on their daily lives. Its relative prevalence and symptoms represent a significant public health problem in Spain. Prospective follow up studies indicate an unsatisfactory clinical evolution of the disease at 5-7 years [7].

FM's etiopathogenesis is unknown however, it is considered to be multifactorial, and to also include epigenetic factors which lead to a persistent alteration in pain regulation systems and central nociceptive hyperexcitability, together with a decrease in the modulation of response inhibition activity and pain control [8].

According to a study by the Spanish Society of Rheumatology (EPISER), it is calculated that in Spain between 2 and $4 \%$ of the population suffer from FM, $0.34 \%$ in Catalonia, with greater prevalence among women than men [9]. It is commonly encountered in clinical practice, representing 5 to $8 \%$ of primary care consultations and between 10 and $20 \%$ of specialist rheumatology consultations. Direct Primary Care costs for patient/year, broken down into its components (medical visits, referrals to referral specialists, complementary tests, and pharmaceutical prescription) were higher in all cases in the FM group. This may indicate that, despite the uncertainty surrounding FM, we should try to avoid delay in diagnosis for patients, which is both costly and inefficient for the health system [10]. Pain is a common symptom, with double the prevalence in women compared to men, especially in those aged over 40 years [11].

FM is recognized as a disease which causes significant morbidity throughout the world. Studies carried out in various countries estimate a prevalence of FM in the general population of between $0.5 \%$ and $5 \%$ [12]: In the United States the prevalence was found to be $2 \%$, lower in men than in women ( $0.5 \%$ and $3.4 \%$ respectively) [13]; Canada $3.3 \%$ with a female to male ration of three to one [14], Italy $2.2 \%$, Sweden $2.5 \%$, Finland $0.8 \%$, Denmark $0.7 \%$, France $1.4 \%$ and Portugal 3.6\% [15-17]. 
Compared with other rheumatological patients, those affected by FM make greater use of healthcare services, with an average of 9-12 consultations per year [18], make greater use of alternative therapies and incur higher economic costs per patient due to absences from work. In addition, patients with FM undergo a greater number of surgical procedures and exhibit a greater number of other rheumatic diseases, such as rheumatoid arthritis, psoriatic arthritis and systemic lupus erythematosus, as well as gastrointestinal disorders and arterial hypertension, neuropathic pain, spinal pain, respiratory infections, sleep disturbances, and mood disorders [19].

The aim of this study was to determine the prevalence and distribution of the incidence of individuals diagnosed with fibromyalgia between 2010 and 2017 based on sex, age and MEDEA deprivation index in Catalonia. Additionally, we wanted to evaluate whether any difference between genders and ages.

\section{Methods}

\subsection{Design and participants}

A retrospective observational study of the population of Catalonia between 2010-2017 both included. The scope of study were the 283 primary care teams (PCT) managed by the Catalan Institute of Health (ICS).

Patient data was obtained from the Information System for the Development of Research in Primary Care (SIDIAP) (www.sidiap.org). This clinical database uses data from the primary care service's electronic medical records programme (ECAP) and other complementary data sources which contain individual information on every patient linked to a unique, anonymous identifier .

As can be seen in Fig. 1, in Catalonia (an autonomous community in the northeaster corner of Spain) $6,378,910$ individuals ( $80 \%$ of the population) are registered in the SIDIAP data base. This statistic is of special interest since it is not an estimate based on a sample, but rather the actual total of all active diagnoses in Catalonia. The following were excluded from the study: patients who were covered by the Mutualidad General de Funcionarios Civiles del Estado [the General Civil Servants Mutual Fund] (Muface, Isfass or similar) and those whose demographic variables of age, sex and PAD were incomplete $(n=5,930,060)$. All individuals $\geq 18$ years of age with a diagnosis of FM (code M79.7) according to the International Classification of Diseases (ICD-10) registered in the ECAP electronic medical records programme were included in the study.

\subsection{Data sources}

The demographic variables studied were: sex (female, male), age ( $\geq 18-<75$ years), deprivation index (MEDEA), patients at the end of follow up (alive, dead, transferred), tobacco consumption (non-smoker, smoker, former-smoker, unknown) and alcohol risk (no risk, low risk, high risk, unknown).

The MEDEA deprivation index [20] was used to measure the participants' socioec-onomic level. The index classifies individuals by socioeconomic level according to whether they live in a rural (R) or urban (U) area. In urban areas, the index divides the inhabitants into quartiles where the first (U1) and fifth(U5) quartiles are the least and most disadvantaged areas, respectively [20]. Urban areas are considered to be municipalities with more than 10,000 inhabitants and with a population density greater than 150/km2 [21].

This study was approved by the IDIAP Jordi Gol's Research Ethics Committee (Code P18/081). The data was anonymized and processed with the utmost confidentiality.

\subsection{Statistical analysis}


The data was analysed and processed with $\mathrm{R}$ statistical program, version 3.6.1. The sample was described using absolute frequencies and percentages in the case of categorical variables and with median and quartiles in the case of continuous variables. The cumulative prevalence and incidence of FM diagnosis during 2010 and 2017 were estimated based on sex, age and the MEDEA deprivation index with a 95\% confidence interval. To estimate the annual prevalence, the number of FM cases was divided by the total population for each year. To estimate the annual cumulative incidence, the number of newly diagnosed cases was divided by the number of cases free from FM at the beginning of each year. Finally, a logistic regression model was performed to evaluate the prevalence of FM based on sex, age, year and sex*age, sex*year interactions. The model results are reported as odds ratio (OR) with a 95\% confidence interval.

\section{Results}

\subsection{Sample characteristics}

Although 56,203 patients initially met the inclusion criteria, 105 were excluded because they were under 18 years of age. The final study was carried out with 56,098 patients diagnosed with FM (Fig. 1).

Table 1 shows how the diagnosis of FM is higher in females (95.4\%) compared to males (4.55\%), with a mean age of 53.0 [45.0-61.0] years. More cases of FM were detected in rural areas (17.3\%) than in urban areas where there is an increasing trend depending on the degree of deprivation; the higher the deprivation, the greater the number of cases diagnosed with FM. $12.69 \%$ of those affected engage in high risk alcohol consumption, $24.7 \%$ are active smokers and $10.9 \%$ are former smokers. 
Table 1

Demographic characteristics of patients diagnosed with FM.

\begin{tabular}{|c|c|}
\hline & $\begin{array}{l}\text { Case FM } \\
\mathrm{N}=56.098\end{array}$ \\
\hline \multicolumn{2}{|l|}{ Sex } \\
\hline Female & $53.545(95.4 \%)$ \\
\hline Male & $2.553(4.55 \%)$ \\
\hline Age & $53.1[45.0 ; 61.0]$ \\
\hline \multicolumn{2}{|l|}{ MEDEA } \\
\hline Unknown & $47(0.08 \%)$ \\
\hline Rural & 9,709 (17.3\%) \\
\hline Urban $^{1}$ & 4,331 (7.72\%) \\
\hline U1 & $7,020(12.5 \%)$ \\
\hline U2 & $8,240(14.7 \%)$ \\
\hline U3 & $8,939(15.9 \%)$ \\
\hline U4 & $9,165(16.3 \%)$ \\
\hline U5 & 8,647 (15.4\%) \\
\hline \multicolumn{2}{|c|}{ Patients at follow-up } \\
\hline Alive & $51,602(92.0 \%)$ \\
\hline Dead & $912(1.63 \%)$ \\
\hline Transferred & $3,584(6.39 \%)$ \\
\hline \multicolumn{2}{|l|}{ Alcohol risk } \\
\hline No risk & $25,544(45.5 \%)$ \\
\hline Low risk & $6,854(12.2 \%)$ \\
\hline High risk & $276(0.49 \%)$ \\
\hline Unknown & $23,424(41.8 \%)$ \\
\hline \multicolumn{2}{|c|}{ Tabaco consumption } \\
\hline Non-smoker & $27,937(49.8 \%)$ \\
\hline Smoker & $13.836(24.7 \%)$ \\
\hline Former-smoker & $6,118(10.9 \%)$ \\
\hline Unknown & $8,207(14.6 \%)$ \\
\hline
\end{tabular}

${ }^{1}$ Unclassified urban zone in quintiles (U1-U5)

\subsection{FM prevalence}


The study showed an increase in the annual prevalence of FM (Table 3$)$ over the 7-year study period $(0.4 \%(95 \% \mathrm{Cl}=$ $(0.39 ; 0.40)$ in 2010 and $1.07 \%(95 \% \mathrm{Cl}=(1.06 ; 1.08)$ in 2017$)$. This increase was present for both men and women (from $0.03-0.10 \%$ in men compared to an increase from $0.75-2 \%$ in women), being higher in the latter during the whole study period. The age group between $55-65$ years old presented the highest prevalence, with $1.05 \%$ in 2010 , and $2.46 \%$ in 2017.

Prevalence was largely similar between rural areas and urban areas. However, a relationship was observed between prevalence and deprivation in urban areas; the greater the deprivation, the greater the prevalence.

\subsection{Incidence of FM}

Compared with the growth in prevalence, the incidence of FM diagnosis in the population remained constant during the period under study $(0.11 \%(95 \% \mathrm{Cl}=(0.10 ; 0.11)$ in 2010 and $0.10 \%(95 \% \mathrm{Cl}=(0.09 ; 0.10)$ in 2017$)(\mathrm{Table} 4)$.

FM is more prevalent in the female participants than in male participants and among the population aged $45-55$ years and 55-65 years. Nevertheless, in these same age groups, a slight decrease was observed over the study period. No significant differences were observed in the incidence of FM according to the MEDEA socioeconomic index.

\subsection{Age versus year pattern between genders}

We analysed the evolution of prevalence during the study period based on age separating by sex (Fig. 2). In addition, we adjusted a logistic regression model to fitted the prevalence of FM based on sex, age, year and interactions of sex*age and sex*year (Table 2). 
Table 2

Logistic regression model prevalence of FM based on sex, age, year and interactions of sex*age and sex*year

\begin{tabular}{|c|c|c|}
\hline & OR & $95 \% \mathrm{Cl}$ \\
\hline \multicolumn{3}{|l|}{ Sex } \\
\hline Female & 1 & - \\
\hline Male & $<0,001$ & $(0,00 ; 0,00)$ \\
\hline \multicolumn{3}{|l|}{ Age } \\
\hline $18-24$ & 1 & - \\
\hline $25-34$ & 4,41 & $(4,12 ; 4,74)$ \\
\hline $35-44$ & 16,57 & $(15,51 ; 17,72)$ \\
\hline $45-54$ & 46,05 & $(43,13 ; 49,23)$ \\
\hline $55-64$ & 70,21 & $(65,77 ; 75,05)$ \\
\hline $65-74$ & 46,97 & $(43,98 ; 50,22)$ \\
\hline$>=75$ & 11,66 & $(10,91 ; 12,49)$ \\
\hline Year & 1,12 & $(1,12 ; 1,12)$ \\
\hline \multicolumn{3}{|l|}{ Sex*Age } \\
\hline Male*18-24 & 1 & - \\
\hline Male*25-34 & 0,51 & $(0,43 ; 0,62)$ \\
\hline Male*35-44 & 0,37 & $(0,31 ; 0,44)$ \\
\hline Male $45-54$ & 0,28 & $(0,21 ; 0,33)$ \\
\hline Male $\star 55-64$ & 0,23 & $(0,19 ; 0,27)$ \\
\hline Male*65-74 & 0,18 & $(0,15 ; 0,21)$ \\
\hline Male*>=75 & 0,24 & $(0,20 ; 0,29)$ \\
\hline \multicolumn{3}{|l|}{ Sex*Year } \\
\hline Male*Year & 1,01 & $(1,01 ; 1,02)$ \\
\hline
\end{tabular}

A significant effect of sex was observed with an $\mathrm{OR}<0.01,95 \% \mathrm{Cl}=(0.00 ; 0.00))$. A significant effect of age was observed; with respect to individuals aged 18 to 24 years, all categories have an OR greater than 1, especially individuals between 45 and 74 years, and a significant effect of the year was observed with an $\mathrm{OR}=1.12(95 \% \mathrm{Cl}=(1.12 ; 1.12))$ (Table 2).

The interaction between sex and age was significant for all age categories, i.e., the behavior of FM prevalence in different age groups varies by gender. The interaction between sex and year has also been significant, ie the evolution of the prevalence over the period analyzed has been different depending on sex $(\mathrm{OR}=1.01,95 \% \mathrm{Cl}=(1.01 ; 1.02))$ (Table 2). 
Table 3

Cases and prevalence (\%) at overall population and by sex, age and MEDEA index.

\begin{tabular}{|c|c|c|c|c|c|c|c|c|}
\hline & 2010 & 2011 & 2012 & 2013 & 2014 & 2015 & 2016 & 2017 \\
\hline Overall & $\begin{array}{l}21,817 \\
(0.40)\end{array}$ & $\begin{array}{l}27,191 \\
(0.50)\end{array}$ & $\begin{array}{l}31,549 \\
(0.59)\end{array}$ & $\begin{array}{l}35,920 \\
(0.68)\end{array}$ & $\begin{array}{l}40,211 \\
(0.77)\end{array}$ & $\begin{array}{l}43,983 \\
(0.86)\end{array}$ & $\begin{array}{l}47,404 \\
(0.96)\end{array}$ & $\begin{array}{l}50,299 \\
(1.07)\end{array}$ \\
\hline $95 \% \mathrm{Cl}$ & $\begin{array}{l}(0.39 \\
0.40)\end{array}$ & $\begin{array}{l}(0.49 \\
0.51)\end{array}$ & $\begin{array}{l}(0.58 \\
0.59)\end{array}$ & $\begin{array}{l}(0.67 \\
0.69)\end{array}$ & $\begin{array}{l}(0.77 \\
0.78)\end{array}$ & $\begin{array}{l}(0.85 \\
0.87)\end{array}$ & $\begin{array}{l}(0.95 \\
0.97)\end{array}$ & $\begin{array}{l}(1.06, \\
1.08)\end{array}$ \\
\hline \multicolumn{9}{|l|}{ Sex } \\
\hline Female & $\begin{array}{l}20,919 \\
(0.75)\end{array}$ & $\begin{array}{l}26,077 \\
(0.95)\end{array}$ & $\begin{array}{l}30,230 \\
(1.11)\end{array}$ & $\begin{array}{l}34,377 \\
(1.28)\end{array}$ & $\begin{array}{l}38,466 \\
(1.45)\end{array}$ & $\begin{array}{l}42,060 \\
(1.62)\end{array}$ & $\begin{array}{l}45,291 \\
(1.80)\end{array}$ & $\begin{array}{l}48,077 \\
(2.00)\end{array}$ \\
\hline Male & $\begin{array}{l}989 \\
(0.03)\end{array}$ & $\begin{array}{l}1,114 \\
(0.04)\end{array}$ & $\begin{array}{l}1,319 \\
(0.05)\end{array}$ & $\begin{array}{l}1,543 \\
(0.06)\end{array}$ & $\begin{array}{l}1,745 \\
(0.07)\end{array}$ & $\begin{array}{l}1,923 \\
(0.08)\end{array}$ & $\begin{array}{l}2,113 \\
(0.09)\end{array}$ & $\begin{array}{l}2,222 \\
(0.10)\end{array}$ \\
\hline \multicolumn{9}{|l|}{ Age } \\
\hline $18-25$ & $\begin{array}{l}110 \\
(0.02)\end{array}$ & $\begin{array}{l}118 \\
(0.02)\end{array}$ & $\begin{array}{l}119 \\
(0.02)\end{array}$ & $\begin{array}{l}123 \\
(0.03)\end{array}$ & $\begin{array}{l}129 \\
(0.03)\end{array}$ & $\begin{array}{l}144 \\
(0.03)\end{array}$ & $\begin{array}{l}149 \\
(0.04)\end{array}$ & $\begin{array}{l}153 \\
(0.04)\end{array}$ \\
\hline $25-35$ & $\begin{array}{l}937 \\
(0.08)\end{array}$ & $\begin{array}{l}1,060 \\
(0.10)\end{array}$ & $\begin{array}{l}1,077 \\
(0.11)\end{array}$ & $\begin{array}{l}1,113 \\
(0.12)\end{array}$ & $\begin{array}{l}1,109 \\
(0.13)\end{array}$ & $\begin{array}{l}1,081 \\
(0.13)\end{array}$ & $\begin{array}{l}1,061 \\
(0.14)\end{array}$ & $\begin{array}{l}1,014 \\
(0.15)\end{array}$ \\
\hline $35-45$ & $\begin{array}{l}3,310 \\
(0.29)\end{array}$ & $\begin{array}{l}3,896 \\
(0.34)\end{array}$ & $\begin{array}{l}4,278 \\
(0.37)\end{array}$ & $\begin{array}{l}4,702 \\
(0.41)\end{array}$ & $\begin{array}{l}5,004 \\
(0.44)\end{array}$ & $\begin{array}{l}5,219 \\
(0.47)\end{array}$ & $\begin{array}{l}5,313 \\
(0.50)\end{array}$ & $\begin{array}{l}5,323 \\
(0.54)\end{array}$ \\
\hline $45-55$ & $\begin{array}{l}6,822 \\
(0.80)\end{array}$ & $\begin{array}{l}8,312 \\
(0.96)\end{array}$ & $\begin{array}{l}9,400 \\
(1.07)\end{array}$ & $\begin{array}{l}10,175 \\
(1.15)\end{array}$ & $\begin{array}{l}11,062 \\
(1.24)\end{array}$ & $\begin{array}{l}11,656 \\
(1.29)\end{array}$ & $\begin{array}{l}12,233 \\
(1.37)\end{array}$ & $\begin{array}{l}12,611 \\
(1.43)\end{array}$ \\
\hline $55-65$ & $\begin{array}{l}7,040 \\
(1.05)\end{array}$ & $\begin{array}{l}8,881 \\
(1.32)\end{array}$ & $\begin{array}{l}10,395 \\
(1.54)\end{array}$ & $\begin{array}{l}11,866 \\
(1.75)\end{array}$ & $\begin{array}{l}13,291 \\
(1.95)\end{array}$ & $\begin{array}{l}14,666 \\
(2.13)\end{array}$ & $\begin{array}{l}15,800 \\
(2.30)\end{array}$ & $\begin{array}{l}16,674 \\
(2.46)\end{array}$ \\
\hline $65-75$ & $\begin{array}{l}2,837 \\
(0.56)\end{array}$ & $\begin{array}{l}3,809 \\
(0.76)\end{array}$ & $\begin{array}{l}4,805 \\
(0.94)\end{array}$ & $\begin{array}{l}6,100 \\
(1.15)\end{array}$ & $\begin{array}{l}7,396 \\
(1.34)\end{array}$ & $\begin{array}{l}8,412 \\
(1.54)\end{array}$ & $\begin{array}{l}9,536 \\
(1.75)\end{array}$ & $\begin{array}{l}10,758 \\
(1.98)\end{array}$ \\
\hline$\geq 75$ & $\begin{array}{l}761 \\
(0.13)\end{array}$ & $\begin{array}{l}1,115 \\
(0.18)\end{array}$ & $\begin{array}{l}1,475 \\
(0.24)\end{array}$ & $\begin{array}{l}1,841 \\
(0.30)\end{array}$ & $\begin{array}{l}2,220 \\
(0.37)\end{array}$ & $\begin{array}{l}2,805 \\
(0.46)\end{array}$ & $\begin{array}{l}3,312 \\
(0.57)\end{array}$ & $\begin{array}{l}3,766 \\
(0.69)\end{array}$ \\
\hline \multicolumn{9}{|l|}{ MEDEA } \\
\hline Unknown & $31(0.30)$ & $32(0.32)$ & $30(0.34)$ & $32(0.39)$ & $33(0.44)$ & $26(0.39)$ & $25(0.45)$ & $16(0.40)$ \\
\hline Rural & $\begin{array}{l}3,763 \\
(0.40)\end{array}$ & $\begin{array}{l}4,643 \\
(0.50)\end{array}$ & $\begin{array}{l}5,442 \\
(0.59)\end{array}$ & $\begin{array}{l}6,240 \\
(0.68)\end{array}$ & $\begin{array}{l}6,901 \\
(0.77)\end{array}$ & $\begin{array}{l}7,560 \\
(0.85)\end{array}$ & $\begin{array}{l}8,241 \\
(0.94)\end{array}$ & $\begin{array}{l}8,811 \\
(1.04)\end{array}$ \\
\hline $\begin{array}{l}\text { Urban no } \\
\text { clasificate }^{1}\end{array}$ & $\begin{array}{l}1,880 \\
(0.32)\end{array}$ & $\begin{array}{l}2,277 \\
(0.39)\end{array}$ & $\begin{array}{l}2,567 \\
(0.47)\end{array}$ & $\begin{array}{l}2,832 \\
(0.54)\end{array}$ & $\begin{array}{l}3,048 \\
(0.62)\end{array}$ & $\begin{array}{l}3,199 \\
(0.70)\end{array}$ & $\begin{array}{l}3,056 \\
(0.82)\end{array}$ & $\begin{array}{l}2,687 \\
(1.00)\end{array}$ \\
\hline Urban $^{2}$ & $\begin{array}{l}18,023 \\
(0.39)\end{array}$ & $\begin{array}{l}22,516 \\
(0.50)\end{array}$ & $\begin{array}{l}26,077 \\
(0.59)\end{array}$ & $\begin{array}{l}29,648 \\
(0.68)\end{array}$ & $\begin{array}{l}33,277 \\
(0.78)\end{array}$ & $\begin{array}{l}36,397 \\
(0.87)\end{array}$ & $\begin{array}{l}39,138 \\
(0.97)\end{array}$ & $\begin{array}{l}41,472 \\
(1.08)\end{array}$ \\
\hline U1 & $\begin{array}{l}2,830 \\
(0.34)\end{array}$ & $\begin{array}{l}3,529 \\
(0.42)\end{array}$ & $\begin{array}{l}4,131 \\
(0.50)\end{array}$ & $\begin{array}{l}4,687 \\
(0.57)\end{array}$ & $\begin{array}{l}5,244 \\
(0.65)\end{array}$ & $\begin{array}{l}5,680 \\
(0.71)\end{array}$ & $\begin{array}{l}6,001 \\
(0.78)\end{array}$ & $\begin{array}{l}6,372 \\
(0.85)\end{array}$ \\
\hline U2 & $\begin{array}{l}3,231 \\
(0.41)\end{array}$ & $\begin{array}{l}4,020 \\
(0.52)\end{array}$ & $\begin{array}{l}4,654 \\
(0.60)\end{array}$ & $\begin{array}{l}5,343 \\
(0.70)\end{array}$ & $\begin{array}{l}6,014 \\
(0.79)\end{array}$ & $\begin{array}{l}6,603 \\
(0.88)\end{array}$ & $\begin{array}{l}7,171 \\
(0.97)\end{array}$ & $\begin{array}{l}7,686 \\
(1.06)\end{array}$ \\
\hline U3 & $\begin{array}{l}3,460 \\
(0.45)\end{array}$ & $\begin{array}{l}4,355 \\
(0.57)\end{array}$ & $\begin{array}{l}5,048 \\
(0.66)\end{array}$ & $\begin{array}{l}5,742 \\
(0.76)\end{array}$ & $\begin{array}{l}6,464 \\
(0.87)\end{array}$ & $\begin{array}{l}7,083 \\
(0.96)\end{array}$ & $\begin{array}{l}7,728 \\
(1.06)\end{array}$ & $\begin{array}{l}8,324 \\
(1.16)\end{array}$ \\
\hline U4 & $\begin{array}{l}3,519 \\
(0.46)\end{array}$ & $\begin{array}{l}4,423 \\
(0.58)\end{array}$ & $\begin{array}{l}5,131 \\
(0.68)\end{array}$ & $\begin{array}{l}5,836 \\
(0.78)\end{array}$ & $\begin{array}{l}6,568 \\
(0.88)\end{array}$ & $\begin{array}{l}7,209 \\
(0.98)\end{array}$ & $\begin{array}{l}7,872 \\
(1.09)\end{array}$ & $\begin{array}{l}8,448 \\
(1.20)\end{array}$ \\
\hline
\end{tabular}




\begin{tabular}{|lllllllll|}
\hline & 2010 & 2011 & 2012 & 2013 & 2014 & 2015 & 2016 & 2017 \\
\hline U5 & 3,103 & 3,912 & 4,546 & 5,208 & 5,939 & 6,623 & 7,310 & 7,955 \\
& $(0.41)$ & $(0.52)$ & $(0.61)$ & $(0.71)$ & $(0.81)$ & $(0.92)$ & $(1.04)$ & $(1.16)$ \\
\hline
\end{tabular}

${ }^{1}$ unclassified urban zone in quintiles (U1-U5); ${ }^{2}$ Sum of all urban categories (U1 least deprived-U5 most deprived). 
Table 4

New cases and cumulative incidence (\%) for total population and by sex, age and MEDEA index.

\begin{tabular}{|c|c|c|c|c|c|c|c|c|}
\hline & 2010 & 2011 & 2012 & 2013 & 2014 & 2015 & 2016 & 2017 \\
\hline Total & $\begin{array}{l}6,042 \\
(0.11)\end{array}$ & $4,85: 7(0.09)$ & $\begin{array}{l}4,561 \\
(0.09)\end{array}$ & $4,811(0.09)$ & )$^{4,446(0.09}$ & $\begin{array}{l}4342 \\
(0.09)\end{array}$ & $\begin{array}{l}4,143 \\
(0.08)\end{array}$ & $\begin{array}{l}4,526 \\
(0.10)\end{array}$ \\
\hline $95 \% \mathrm{Cl}$ & $\begin{array}{l}(0.10, \\
0.11)\end{array}$ & $(0.08,0.09)$ & $\begin{array}{l}(0.08, \\
0.09)\end{array}$ & $(0.09,0.09)$ & $(0.08,0.09)$ & $\begin{array}{l}(0.08, \\
0.09)\end{array}$ & $\begin{array}{l}(0.08, \\
0.09)\end{array}$ & $\begin{array}{l}(0.09, \\
0.10)\end{array}$ \\
\hline \multicolumn{9}{|l|}{ Sex } \\
\hline Female & $\begin{array}{l}5,787 \\
(0.21)\end{array}$ & $\begin{array}{ll}4,658 & 4,327 \\
(0.17) & (0.16)\end{array}$ & \multicolumn{2}{|c|}{$4,571(0.17)$} & )$^{4114(}$ & )$^{4114(0.16}$ & $(0.16$ & $\begin{array}{l}4,302 \\
(0.18)\end{array}$ \\
\hline Male & $\begin{array}{l}255 \\
(0.01)\end{array}$ & $199(0.01)$ & $\begin{array}{l}234 \\
(0.01)\end{array}$ & $240(0.01)$ & $202(0.01)$ & $228(0.01)$ & $\begin{array}{l}226 \\
(0.01)\end{array}$ & $\begin{array}{l}224 \\
(0.01)\end{array}$ \\
\hline \multicolumn{9}{|l|}{ Age } \\
\hline $18-25$ & $\begin{array}{l}40 \\
(0.01)\end{array}$ & $38(0.01)$ & $\begin{array}{l}33 \\
(0.01)\end{array}$ & $33(0.01)$ & $27(0.01)$ & $34(0.01)$ & $28(0.01)$ & $\begin{array}{l}23 \\
(0.01)\end{array}$ \\
\hline $25-35$ & $\begin{array}{l}326 \\
(0.03)\end{array}$ & $263(0.02)$ & $\begin{array}{l}224 \\
(0.02)\end{array}$ & $269(0.03)$ & $204(0.02)$ & $218(0.03)$ & $\begin{array}{l}200 \\
(0.03)\end{array}$ & $\begin{array}{l}222 \\
(0.03)\end{array}$ \\
\hline $35-45$ & $\begin{array}{l}992 \\
(0.09)\end{array}$ & $873(0.08)$ & $\begin{array}{l}827 \\
(0.07)\end{array}$ & $854(0.07)$ & $811(0.07)$ & $851(0.08)$ & $\begin{array}{l}765 \\
(0.07)\end{array}$ & $\begin{array}{l}822 \\
(0.08)\end{array}$ \\
\hline $45-55$ & $\begin{array}{l}2,012 \\
(0.24)\end{array}$ & $1,627(0.19)$ & $\begin{array}{l}1,492 \\
(0.17)\end{array}$ & $1,538(0.17)$ & $1,480(0.17)$ & $\begin{array}{l}1419 \\
(0.16)\end{array}$ & $\begin{array}{l}1,449 \\
(0.16)\end{array}$ & $\begin{array}{l}1,518 \\
(0.17)\end{array}$ \\
\hline $55-65$ & $\begin{array}{l}1,820 \\
(0.27)\end{array}$ & $1,368(0.21)$ & $\begin{array}{l}1,289 \\
(0.19)\end{array}$ & $1,336(0.20)$ & $1,150(0.17)$ & $\begin{array}{l}1130 \\
(0.17)\end{array}$ & $\begin{array}{l}1,072 \\
(0.16)\end{array}$ & $\begin{array}{l}1,185 \\
(0.18)\end{array}$ \\
\hline $65-75$ & $\begin{array}{l}670 \\
(0.13)\end{array}$ & $530(0.11)$ & $\begin{array}{l}542 \\
(0.11)\end{array}$ & $603(0.12)$ & $607(0.11)$ & $504(0.09)$ & $\begin{array}{l}481 \\
(0.09)\end{array}$ & $\begin{array}{l}560 \\
(0.10)\end{array}$ \\
\hline$\geq 75$ & $\begin{array}{l}182 \\
(0.03)\end{array}$ & $158(0.03)$ & $\begin{array}{l}154 \\
(0.03)\end{array}$ & $178(0.03)$ & $167(0.03)$ & $186(0.03)$ & $\begin{array}{l}148 \\
(0.03)\end{array}$ & $\begin{array}{l}196 \\
(0.03)\end{array}$ \\
\hline \multicolumn{9}{|l|}{ MEDEA } \\
\hline Unknown & $\begin{array}{l}8 \\
(0.08)\end{array}$ & $0(0.00)$ & $\begin{array}{l}3 \\
(0.03)\end{array}$ & $4(0.0)$ & $3(0.04)$ & $2(0.03)$ & $3(0.05)$ & $\begin{array}{l}2 \\
(0.04)\end{array}$ \\
\hline Rural & $\begin{array}{l}1,033 \\
(0.11)\end{array}$ & $832(0.09)$ & $\begin{array}{l}801 \\
(0.09)\end{array}$ & $822(0.09)$ & $729(0.08)$ & $739(0.08)$ & $\begin{array}{l}752 \\
(0.09)\end{array}$ & $\begin{array}{l}836 \\
(0.10)\end{array}$ \\
\hline $\begin{array}{l}\text { Urban not } \\
\text { classified }^{1}\end{array}$ & $\begin{array}{l}492 \\
(0.08)\end{array}$ & $391(0.07)$ & $\begin{array}{l}348 \\
(0.06)\end{array}$ & $348(0.07)$ & $328(0.07)$ & $310(0.07)$ & $\begin{array}{l}270 \\
(0.07)\end{array}$ & $\begin{array}{l}256 \\
(0.08)\end{array}$ \\
\hline Urban $^{2}$ & $\begin{array}{l}5,001 \\
(0.11)\end{array}$ & 4,025 (0.09) & $\begin{array}{l}3,757 \\
(0.08)\end{array}$ & 3,985 (0.09) & $3,714(0.09)$ & $\begin{array}{l}3,601 \\
(0.09)\end{array}$ & $\begin{array}{l}3,388 \\
(0.08)\end{array}$ & $\begin{array}{l}3,688 \\
(0.09)\end{array}$ \\
\hline U1 & $\begin{array}{l}766 \\
(0.09)\end{array}$ & $638(0.08)$ & $\begin{array}{l}595 \\
(0.07)\end{array}$ & $607(0.07)$ & $554(0.07)$ & $476(0.06)$ & $\begin{array}{l}461 \\
(0.06)\end{array}$ & $\begin{array}{l}510 \\
(0.07)\end{array}$ \\
\hline U2 & $\begin{array}{l}909 \\
(0.12)\end{array}$ & $696(0.09)$ & $\begin{array}{l}677 \\
(0.09)\end{array}$ & $739(0.10)$ & $664(0.09)$ & $642(0.09)$ & $\begin{array}{l}568 \\
(0.08)\end{array}$ & $\begin{array}{l}644 \\
(0.09)\end{array}$ \\
\hline U3 & $\begin{array}{l}965 \\
(0.13)\end{array}$ & $791(0.10)$ & $\begin{array}{l}711 \\
(0.09)\end{array}$ & $780(0.10)$ & $703(0.09)$ & $704(0.10)$ & $\begin{array}{l}647 \\
(0.09)\end{array}$ & $\begin{array}{l}721 \\
(0.10)\end{array}$ \\
\hline
\end{tabular}




\begin{tabular}{|lllllllll|}
\hline & 2010 & 2011 & 2012 & 2013 & 2014 & 2015 & 2016 & 2017 \\
\hline U4 & 992 & $806(0.11)$ & $\begin{array}{l}731 \\
(0.10)\end{array}$ & $766(0.10)$ & $736(0.10)$ & $729(0.10)$ & 707 & 740 \\
& $(0.13)$ & & & & & $(0.10)$ & $(0.10)$ \\
\hline U5 & $\begin{array}{l}877 \\
(0.11)\end{array}$ & $703(0.09)$ & $\begin{array}{l}695 \\
(0.09)\end{array}$ & $745(0.10)$ & $729(0.10)$ & $740(0.10)$ & 735 & $\begin{array}{l}817 \\
(0.11)\end{array}$ \\
& & & & & $(0.12)$ \\
\hline
\end{tabular}

${ }^{1}$ Unclassified urban zone in quintiles (U1-U5); ${ }^{2}$ Sum of all urban categories (U1 least deprived-U5 most deprived

\section{Discussion}

FM is the rheumatic disease which most effects the quality of life of patients since they report a significant impact on their physical and intellectual capacity, personal relationships, etc. [22] In the majority of cases patients are unable to work and perceive their health to be poor. A diagnosis may not be made until many years after the initial onset of symptoms FM is a chronic disease which affects the functional capacity of patients to such an extent that they may be unable to carry out some of their usual daily activities [23]. It is a prevalent disease and our work corroborates many of the points that have been shown in other epidemiological studies, such the wider prevalence in women and the growing trend of cases diagnosed at all ages.

Following a literature review, we were unable to find similar studies which have been carried out in Catalonia with the same sample size in order to carry out a comparison. However, in general the results are in consonance with studies conducted in other countries. The EPISER study on rheumatic diseases [24] establishes a prevalence of FM in Spain of $2.4 \%$, with a higher prevalence in females than males and an upward trend over time. These results largely coincide with those in our study: in 2010 the prevalence was $0.75 \%$ in women and $0.03 \%$ in men, while in 2017 it stood at $2 \%$ in women and $0.10 \%$ in men. In line with this, in our cohort studied we found that the diagnosis of FM was less common in male than female. In addition, several studies pointed out to a different pain sensibility threshold and associated factors to subjective perception to pain in both sex. Female generally exhibit higher sensitivity to noxious stimuli not only from mechanical pressure, but also from electric, thermal, ischemic, and cold stimuli [25]. It is plausible that complex biological factors from hormonal influences and psychosociocultural factors including sex expectations may influence too. Sociocultural beliefs about femininity and masculinity also appear to be an important determinant of pain responses among sexes as pain expression is generally more socially acceptable among women, an effect which may lead to biased reporting of pain. In a study [26], both men and women believed that men are less willing to report pain than woman. Such gender role expectations may contribute to sex differences when experimenting pain [27]. Therefore, we cannot exclude that the existence of other cultural, socioeconomic factors or differences between both sexes in terms of the perception of the disease, behavior and attitudes towards health services, may explain this lower prevalence of the disease in men [28]. Another study confirms that males with FM tend to endure pain for longer periods of time than females before seeking treatment. Unfortunately, there is still a paucity of evidence on clinical characterization and treatment options when FM occurs in males. With respect to age, the highest prevalence in our study was found in the 55-65 and 55-65 age groups, with the latter having the highest prevalence. These results concur with the EPISER study in which the group with the highest prevalence was aged 40-59 years. In the EPIFFAC study [29] the mean age of its sample of 325 people was 52 and a study carried out in the United States found the highest prevalence in the group aged 50-59 years.

In Catalonia, a study was carried out between 2011-2013 on patients with fibromyalgia in Primary Care Centres which calculated the mean age of the sample to be 55 years. $97.8 \%$ of patients treated were women while $2.2 \%$ were men [30]. These results coincide with the results obtained in this study, as the most prevalent age is found between 55 and 64 years in both women and men.It is worth highlighting the prevalence of FM in rural areas. 
Although the published literature has not indicated whether a rural environment can be beneficial or protective with regard to FM compared to an urban one, in our results there is a slightly higher percentage of FM in rural areas and a slight positive correlation with the deprivation index in urban areas [19]. It is also observed that the prevalence of FM has been slightly higher in individuals in areas with greater deprivation than individuals in areas with less deprivation.

The consumption of substances such as alcohol and tobacco, could be associated with an FM diagnosis, since some studies show an intake of alcohol and tobacco ( $41.4 \%$ and $38.5 \%$, respectively) above the average of the general population (19\% and 36\%) [31]. However, in our sample only $14.2 \%$ of patients diagnosed with FM have low-risk alcohol consumption and $0.49 \%$ have a high risk, $49.8 \%$ are non-smokers and $24 \%$ are smokers."

FM is not a disease associated with high mortality rates, but it does have significant repercussions on direct and indirect healthcare costs [32]. Over the 7 year study period, $92 \%$ of the individuals diagnosed were still alive, $1.63 \%$ had died and the remainder had left the Catalan health system (6.39\%).

The results of our study may contribute to clinical practice and the review and planning of new protocols in primary care to more effectively address people diagnosed with FM., in addition

\subsection{Study limitations}

Some of FM's symptoms can be confused with those of other rheumatic diseases and this, in turn, can lead to incorrect coding of the diagnosis of FM, We propose to increase diagnostic adequacy by referring patients to specialized FM units to confirm the diagnosis. This study cannot be extrapolated to other countries since it has been conducted in a region of Spain.

\section{Conclusions}

FM is an increasingly prevalent disease in Catalonia with a prevalence increasing from $0.40 \%$ in 2010 to $1.07 \%$ in 2017. The profile with the highest prevalence was women aged between 55 and - 65 with a high deprivation index (MEDEA U4). Few studies have been conducted in Catalonian. More research is required to investigate the behaviour, development and consequences of the disease.

\section{Declarations}

\section{Ethical approval and consent to participate}

This study was approved by the IDIAP Jordi Gol Research Ethics Committee (CEI) and the SIDIAP Scientific Committee Code (P18/081). The data from the SIDIAP database were anonymized, with a null identification risk, in accordance with Organic Law 15/1999, of December 13.

In accordance with the Declaration of Helsinki, we confirm that all methods were carried out in accordance with relevant guidelines and regulations. Furthermore, we confirm that informed consent was obtained from all subjects and/or their legal guardian(s).

\section{Consent for publication}

Not applicable. 


\section{Data Availability Statement}

The data was extracted from the Information System for Research in Primary Care (SIDIAP). These data are not publicly available, and restrictions apply to the availability of the data used for the current study. These data are available upon reasonable request addressed to the corresponding author.

\section{Competing interests}

The authors declare that they have no competing interests.

\section{Funding:}

This work was funded by the SIDIAP (Information System for Rersearch in Primary Care).

\section{Author Contributions:}

Conceptualization, GSV, JVA; Data curation, QMC; Formal analysis, QMC ; Funding acquisition, GSV; Investigation, GSV, JVA; Methodology, GSV; Resources, GSV; Software, QMC; Supervision, GSV and QMC; Visualization, JVA; Writing original draft, GSV; Writing - review \& editing, JVA.

\section{Acknowledgements}

We would like to thank the collaboration of the Institut Universitari per a la Recerca a l'Atenció Primària de Salut Jordi Gol i Gurina (IDIAPJGol) and the Unitat Recerca de Catalunya Central of the Institut Català de la Salut, for their support whenever it is been required

\section{Autors' information}

Not applicable

\section{References}

1. International Association for Study of Pain. The subcommittee on taxonomy. Classification of chronic pain. Description of chronic pain syndromes and definitions of pain terms. Pain Suppl 1986;3:S1-226.

2. Wolfe F, Hawley DJ, Wilson K. The prevalence and meaning of fatigue in rheumatic disease. J Rheumatol. 1996 Aug;23(8):1407-17.

3. White KP, Speechley M, Harth M, Ostbye T. The London Fibromyalgia Epidemiology Study: comparing the demographic and clinical characteristics in 100 random community cases of fibromyalgia versus controls. $J$ Rheumatol. 1999 Jul;26(7):1577-85.

4. Panton LB, Kingsley JD, Toole T, Cress ME, Abboud G, Sirithienthad P, Mathis R, McMillan V. A comparison of physical functional performance and strength in women with fibromyalgia, age and weight matched controls, and older women who are healthy. Phys Ther. 2006 Nov;86(11):1479-88.

5. Mannerkorpi K, Burckhardt CS, Bjelle A. Physical performance characteristics of women with fibromyalgia. Arthritis Care Res. 1994 Sep;7(3):123-9. 
6. Naji A, Arab P. Prevalence of fibromyalgia syndrome in patients referring to Shariati hospital, Tehran. Tehran Univ Med J. 2006; 64 (10):60-66.

7. White KP, Nelson WR, Harth M, Ostbye T, Speechley M. Does the label "fibromyalgia" alter health status, function and health service utilization? A prospective, within group comparison in a community cohort of adults with chronic widespread pain. Arthritis Rheum. 2002;47:260-5.

8. Craig AD. A new view of pain as a homeostatic emotion. Trends Neurosci 2003;26:303-7.

9. Sauch Valmaña G, Vidal-Alaball J, Poch PR, et al. Effects of a Physical Exercise Program on Patients Affected with Fibromyalgia. Journal of Primary Care \& Community Health. January 2020. doi:10.1177/2150132720965071

10. Sicras-Mainar A, Blanca-Tamayo M, Navarro-Artieda R, Rejas-Gutiérrez J. Perfil de uso de recursos y costes en pacientes que demandan atención por fibromialgia o trastorno de ansiedad generalizada en el ámbito de la atención primaria de salud. Aten Primaria. 2009; 41(2): 77-84.

11. Mansfield KE, Sim J, Jordan JL, Jordan KP. A systematic review and meta-analysis of the prevalence of chronic widespread pain in the general population. Pain. 2016 Jan;157(1):55-64. doi: 10.1097/j.pain.0000000000000314.

12. White KP, Harth M. Classification, epidemiology, and natural history of fibromyalgia. Curr Pain Headache Rep 2001;5:320-9.

13. Lawrence RC, Helmick CG, Arnett FC, Deyo RA, Felson DT, Giannini EH, et al. Estimates of the prevalence of arthritis and selected musculoskeletal disorders in the United States. Arthritis Rheum 1998;41:778-99.

14. White KP, Speechley M, Harth M, Ostbye T. The London Fibromyalgia Epidemiology Study: the prevalence of fibromyalgia syndrome in London, Ontario. J Rheumatol 1999;26:1570-6.

15. Salaffi F, De Angelis R, Grassi W; MArche Pain Prevalence; INvestigation Group (MAPPING) study. Prevalence of musculoskeletal conditions in an Italian population sample: results of a regional community based study. I. The MAPPING study. Clin Exp Rheumatol. 2005 Nov-Dec;23(6):819-28.

16. Cöster L, Kendall S, Gerdle B, Henriksson C, Henriksson KG,Bengtsson A. Chronic widespread musculoskeletal pain. A comparison of those who meet criteria for fibromyalgia and those who do not. Eur J Pain 2008;12:600-10.

17. Branco JC, Bannwarth B, Failde I, Abello Carbonell J, Blotman F, Spaeth M, Saraiva F, Nacci F, Thomas E, Caubère JP, Le Lay K, Taieb C, Matucci Cerinic M. Prevalence of fibromyalgia: a survey in five European countries. Semin Arthritis Rheum. 2010 Jun;39(6):448-53. doi: 10.1016/j.semarthrit.2008.12.003.

18. Wolfe F. The relation between tender points and fibromyalgia symptom variables: evidence that fibromyalgia is not a discrete disorder in the clinic. Ann Rheum Dis. 1997;56:268-71

19. Garcia-Gil M, Elorza JM, Banque M, et al. Linking of primary care records to census data to study the association between socioeconomic status and cancer incidence in Southern Europe: a nation-wide ecological study. PLoS One. 2014;9(10):e109706.

20. Domínguez-Berjón M. Felícitas, Borrell Carme, Cano-Serral Gemma, Esnaola Santiago, Nolasco Andreu, Pasarín M. Isabel et al. Construcción de un índice de privación a partir de datos censales en grandes ciudades españolas: (Proyecto MEDEA). Gac Sanit [Internet]. 2008 Jun [Accessed 11 Feb 2020];22(3):179-187. Retrieved from en:http://scielo.isciii.es/scielo.php?script=sci_arttext\&pid=S021391112008000300002\&lng=es.

21. Garcia-Gil M, Hermosilla E, Prieto-Alhambra D, Fina F, Rosell M, et al. (2011) Construction and validation of a scoring system for selection of high-quality data in a Spanish population primary care database (SIDIAP). Inf Prim Care.19(3): 135-45.

22. Programa de desarrollo rural de Catalunya. Período de programación 2007-2013. Generalitat de Catalunya. Departament d’Agricultura, Ramaderia, Pesca, Alimentació i Medi Natural. Decisión C (2008) 702. Seventh Edition, May 2011. 
23. The relationship between chronotype, sleep disturbance, severity of fibromyalgia, and quality of life in patients with fibromyalgia. Türkoğlu G, Selvi Y. Chronobiol Int.2020;37:68-81.

24. Boonen A, Van Den Heuvel R, Van Tubergen A, et al. Large differences in cost of illness and wellbeing between patients with fibromyalgia, chronic low back pain or ankylosing spon dylitis. Ann Rheum Dis 2005; 64:396-402

25. Mas AJ, Carmona L, Valverde M, Ribas B; EPISER Study Group. Prevalence and impact of fibromyalgia on function and quality of life in individuals from the general population: results from a nationwide study in Spain. Clin Exp Rheumatol. 2008 Jul-Aug;26(4):519-26.

26. Yunus MB, Inanici F, Aldag JC, Mangold RF. Fibromyalgia in men: comparison of clinical features with women. J Rheumatol. 2000; 27(2):485-490.

27. Conversano C, Ciacchini R, Orrù G, Bazzichi ML, Gemignani A, Miniati M. Gender differences on psychological factors in fibromyalgia: a systematic review on the male experience. Clin Exp Rheumatol. 2021 May-Jun;39 Suppl 130(3):174-185.

28. Wise EA, Price DD, Myers CD, Heft MW, Robinson ME. Gender role expectations of pain: relationship to experimental pain perception. Pain. 2002;96:335.

29. Conversano C, Ciacchini R, Orrù G, Bazzichi ML, Gemignani A, Miniati M. Gender differences on psychological factors in fibromyalgia: a systematic review on the male experience. Clin Exp Rheumatol. 2021 May-Jun;39 Suppl 130(3):174-185.

30. Collado A, Gómez E, Coscolla R, Sunyol R, Solé E, Rivera J et al. Work, family and social environment in patients with Fibromyalgia in Spain: an epidemiological study: EPIFFAC study. BMC Health Serv Res. 2014; 14: 513

31. Mur Martí T, Llordés Llordés M, Custal Jordà M, López Juan G, Martínez Pardo S. Perfil de pacientes con fibromialgia que acuden a los centros de atención primaria en Terrassa. Reumatol Clin. 2016 Jun 28. pii: S1699$258 \times(16) 30052-3$.

32. Prados G, Miró E, Martínez MP, Sánchez Al, López S, Sáez G. Fibromyalgia: gender differences and sleep-disordered breathing. Clin Exp Rheumatol. 2013;31(6 suppl 79):S102-S110.

33. Walitt B, Nahin RL, Katz RS, Bergman MJ, Wolfe F. The prevalence and characteristics of fibromyalgia in the 2012 National Health Interview Survey. PLoS One. 2015; 10(9).

\section{Figures}


All inhabitants of Catalonia (SIDIAP) aged $\geq 18$ years on December 31, 2017

$(\mathrm{N}=6378910)$

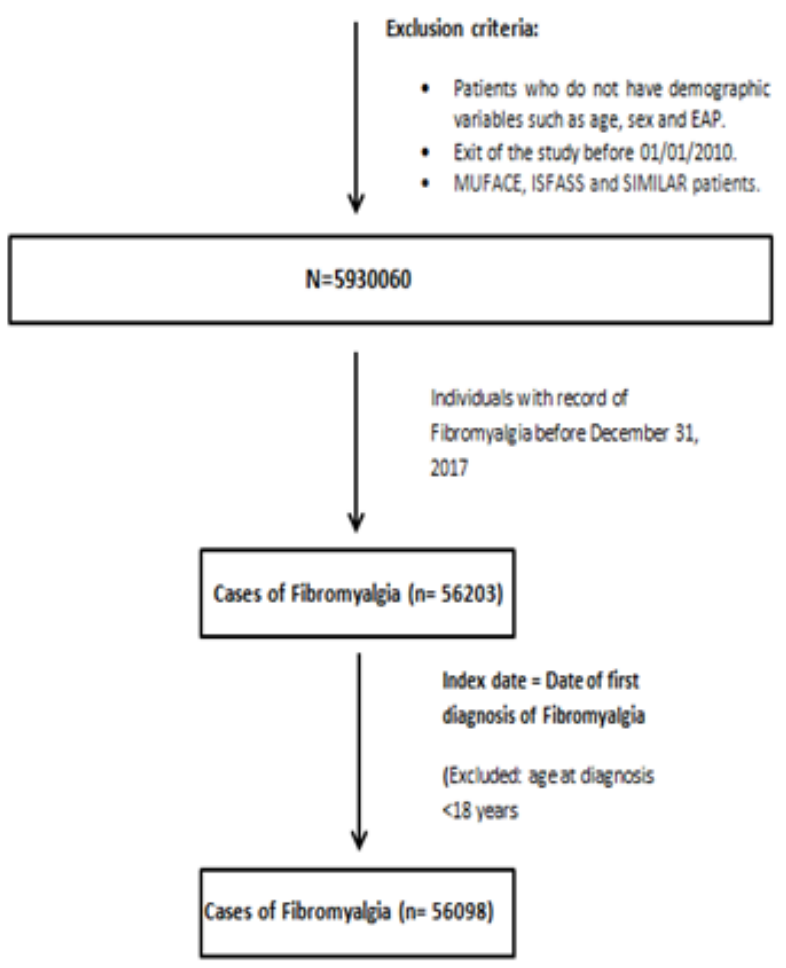

\section{Figure 1}

Flowchart of patient selection. 


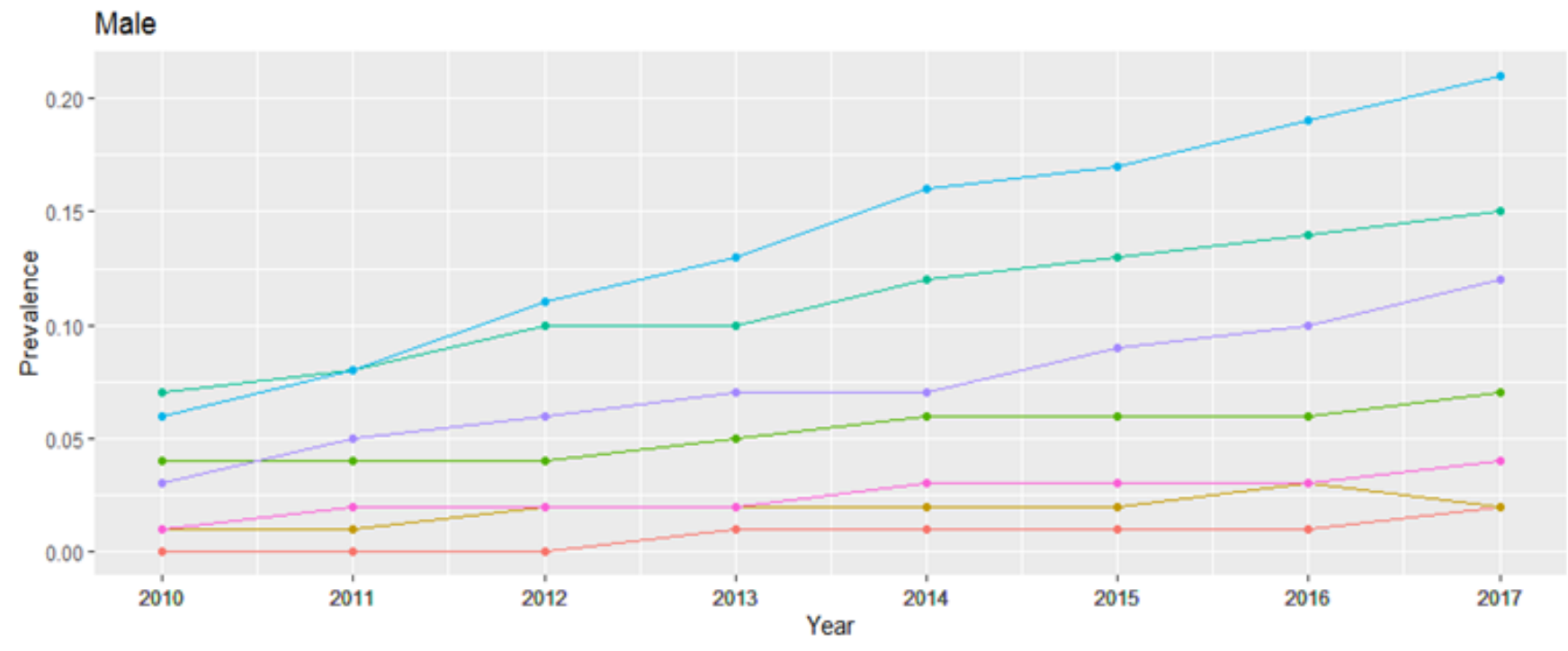

Age

- 1. 18-24

- 2.25-34

- 3. 35-44

- 4. 45-54

- $5.55-64$

- 6.65-74

- $7 .>=75$

Female

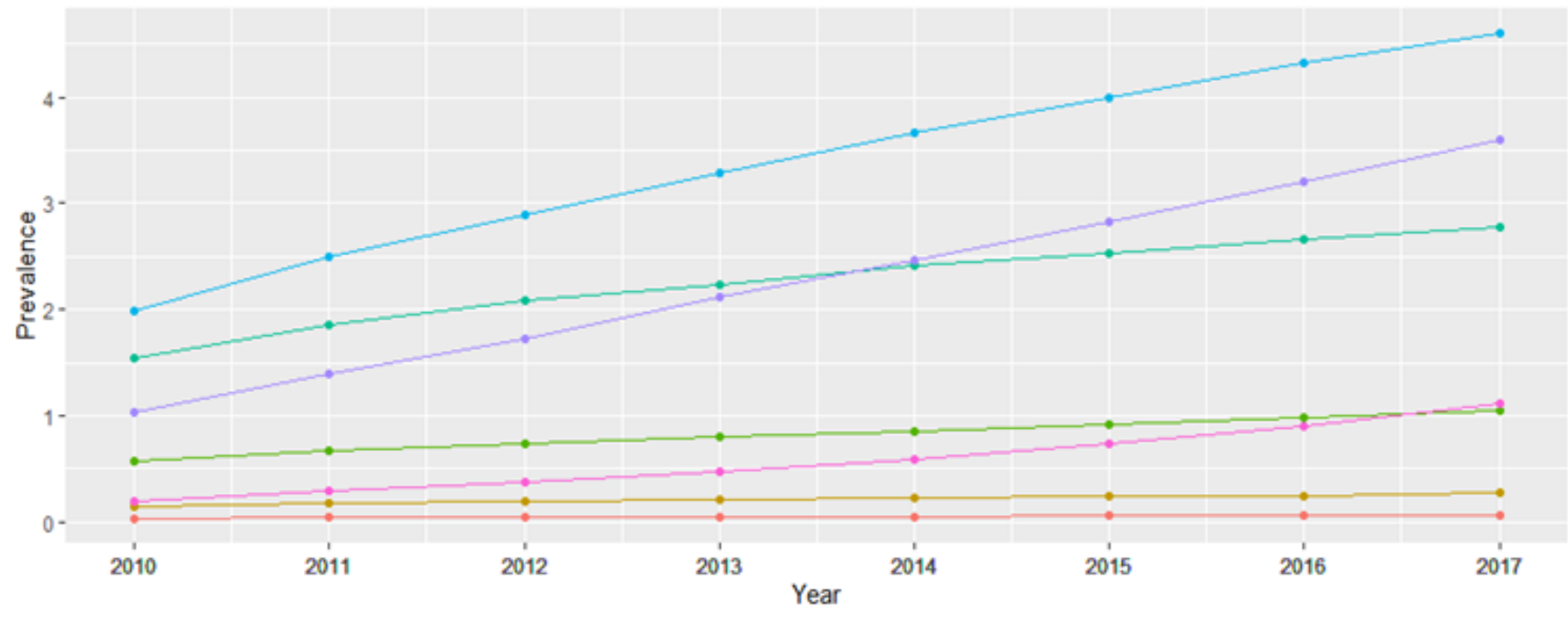

Age

$\rightarrow 1.18-24$

- 2. 25-34

- 3. 35-44

- 4. 45-54

- 5.55-64

$\rightarrow 6.65-74$

$\rightarrow-7 .>=75$

\section{Figure 2}

Prevalence during the study period based on age separating by sex. 